\title{
Frozen to death? Detection of comet Hale-Bopp at 30.7 AU (Research Note)
}

\author{
Gy. M. Szabó ${ }^{1,2,3}$, K. Sárneczky ${ }^{1}$, and L. L. Kiss ${ }^{1,4}$ \\ 1 Konkoly Observatory, Konkoly-Thege Miklós út 15-17, 1121 Budapest, Hungary \\ e-mail: szgy@konkoly.hu \\ 2 Eötvös Fellow at the Astronomy Dept., University of Texas at Austin, 78712 TX, USA \\ 3 Dept. of Exp. Physics \& Astronomical Observatory, University of Szeged, 6720 Szeged, Hungary \\ ${ }^{4}$ Sydney Institute for Astronomy, School of Physics, University of Sydney, NSW 2006, Australia
}

Received 27 February 2011 / Accepted 21 April 2011

\section{ABSTRACT}

\begin{abstract}
Context. Comet Hale-Bopp (C/1995 O1) has been the most interesting comet ever encountered by modern astronomy, which continued to display significant activity at a solar distance of $25.7 \mathrm{AU}$. It is unclear when and how this activity will finally cease.

Aims. We present new observations with the ESO $2.2 \mathrm{~m}$ telescope at La Silla to monitor the activity of Hale-Bopp at 30.7 AU solar distance.

Methods. On 2010-12-04, 26 CCD images were taken with 180 s exposure times for photometry and morphology.

Results. The comet was detected in $R$ and had a total brightness of $23.3 \pm 0.2$, which corresponds to an absolute brightness of $R(1,1,0)=8$. 3 . The profile of the coma was star-like at a seeing of $1^{\prime \prime} .9$, without any evidence of a coma or tail extending farther than $2^{\prime \prime} .5$ (=55000 km in projection) and exceeding $26.5 \mathrm{mag} / \mathrm{arcs}^{2}$ surface brightness. The measured total brightness corresponds to a relative total reflecting surface, $a_{R} C$, of $485 \mathrm{~km}^{2}$, nine times less than three years before. The calculated $a_{R} C$ value would imply a nucleus with $60-65 \mathrm{~km}$ radius assuming $4 \%$ albedo. This size estimate contradicts significantly the previous results scattering around $35 \mathrm{~km}$.

Conclusions. We propose that the comet may still be in a low level of activity, despite the lack of a prominent coma. Alternatively, if the nucleus is already dormant, the albedo should be as high as 13\%, assuming a radius of $35 \mathrm{~km}$. With this observation, Hale-Bopp has become the most distant comet ever observed, far beyond the orbit of Neptune.
\end{abstract}

Key words. comets: individual: Hale-Bopp - techniques: photometric

\section{Introduction}

According to the accepted paradigm of cometary activity, matter production decreases rapidly after the significant decrease of water sublimation at 3 AU (Fernández 2005). A few exceptional comets, however, have displayed activity far beyond 3 AU (e.g. Lowry et al. 1999; Lowry \& Fitzsimmons 2001, 2005; Lowry \& Wiessman 2003; Snodgrass et al. 2006, 2008; Mazzotta Epifani et al. 2006, 2007). There are known examples of active long-period comets (Szabó et al. 2001a, 2002; Meech et al. 2004; Mazzotta Epifani et al. 2009; Ivanova et al. 2011) and Centaur objects (e.g. Meech et al. 1997; Rousselot 2008; Jewitt 2009). The exact location of the snow line is very important in understanding the water reservoirs in the Solar System, hence also the structure and evolution of extrasolar systems, too.

In Szabó et al. (2008), we demonstrated that C/1995 O1 (Hale-Bopp) was the most distant active comet ever observed, with a prominent coma at 25.7 AU solar distance. This surprising observation raised the question of when this activity will cease. Observing Hale-Bopp in a completely frozen state would also be extremely important because a thick coma was constantly present during the entire apparition. The coma obscured the nucleus, which was not observed directly. Lacking photometric data of the bare nucleus, its size, one of the most important input parameters in activity models remained uncertain.

Here we present new observations of Hale-Bopp at a 30.7 AU solar distance. In late 2010, the comet exhibited evidence of the cessation of the matter production. In this paper, we present a photometrical and morphological analysis of these observations.

\section{Observations}

We performed new $R_{C}$ filtered observations with the $2.2 \mathrm{~m}$ ESO telescope at the La Silla site in Chile, on December 4, 2010, with sidereal tracking. We took $26 \times 180 \mathrm{~s}$ exposures with an image scale of $0.24 /$ pixel. The seeing was $\approx 1^{\prime \prime} .9$, mostly because of the airmass of 1.9-2.1, which was caused by the comet's unfavourable celestial position close to the Southern pole.

The images were corrected in a standard fashion, including bias and flat field correction. We aligned and co-added the images by fitting a coordinate grid to the stars, yielding a "star field" image for photometric calibrations. The images were then re-aligned with respect to the proper motion of the comet, to get untrailed "comet" images. In this step, the MPC ephemerides at the time of each observation were used to match the individual frames. Figure 1 shows the "comet" images on December 4, 2010, after co-adding the images taken during the time intervals 5:41-6:48 UT (top panel), and 7:08-8:10 UT (lower panel). The proper motion of the comet confirms its identification.

Hale-Bopp was detected very slightly northwest of the positions predicted for the La Silla site at the time of observations 

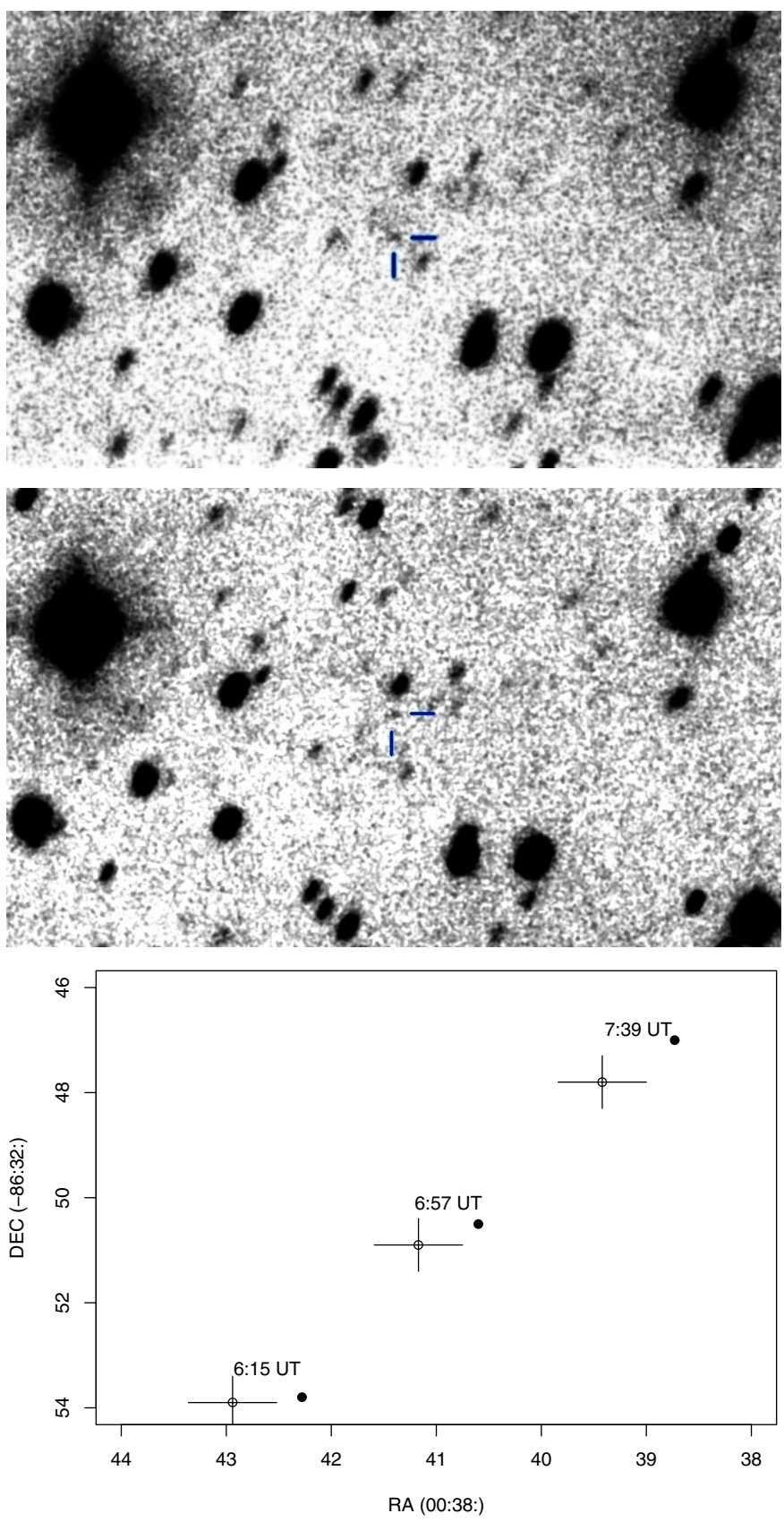

Fig. 1. Upper images: comet Hale-Bopp at 30.7 AU solar distance, on December 4, 2010. The field of view is $2.67 \times 1.57$; north is up, east is to the left. The brightest star in the image is 2MASS J003951208632270, which has an $R$ magnitude of $12.93 \pm 0.06$. Lowest panel: comparison of the measured astrometric positions (open dots with errors) and the JPL predictions (solid dots). Error bars denote the 2-sigma level confidence intervals of the measured positions.

(Table 1, astrometry part; Fig. 1 lowest panel). The mean deviations and their rms scatter in right ascension and declination are $0.59 \pm 0.06$ and $0.25 \pm 0.20$, respectively. The confidence interval of the measured positions was tested by astrometry of artificial sources: with ADDSTAR routine in IRAF, we added 18 artificial comets to the image with the same PSF and brightness as the comet image itself. Because of the noise and sampling, the astrometry of these artificial comets resulted in slightly different positions than where they were inserted. The standard error in these differences was found to be 0.23 in both coordinates, which can be considered as the 1-sigma confidence level error
Table 1. Ephemerides and astronetry of Hale-Bopp on 2010-12-04.

\begin{tabular}{|c|c|c|c|c|c|}
\hline \multicolumn{6}{|c|}{ Ephemerides } \\
\hline$\lambda\left[^{\circ}\right]$ & $\beta\left[^{\circ}\right]$ & $R[\mathrm{AU}]$ & $\Delta[\mathrm{AU}]$ & $E\left[^{\circ}\right]$ & $\alpha\left[^{\circ}\right]$ \\
\hline 278.8 & -66.9 & 30.7 & 31.0 & 69.5 & 1.8 \\
\hline \multicolumn{6}{|c|}{ Astrometry } \\
\hline \multirow{2}{*}{\multicolumn{2}{|c|}{ UT }} & \multicolumn{2}{|c|}{$\mathrm{RA}\left(00^{\mathrm{h}} 38^{\mathrm{m}}\right)$} & \multicolumn{2}{|c|}{$\operatorname{Dec}\left(-86^{\circ} 32^{\prime}\right)$} \\
\hline & & Pred. & Meas. & Pred. & Meas. \\
\hline \multicolumn{2}{|c|}{$6: 15$} & 42.94 & $42^{\mathrm{s}} .28$ & $53{ }^{\prime \prime} 9$ & $533^{\prime \prime} 8$ \\
\hline \multicolumn{2}{|c|}{$6: 57$} & $41^{\mathrm{s}} .17$ & 40.60 & 50.9 & 50,5 \\
\hline \multicolumn{2}{|c|}{$7: 39$} & 39.42 & 38.73 & $47 ! .8$ & $47{ }^{\prime \prime} 0$ \\
\hline
\end{tabular}

in the measured positions of Hale-Bopp. NASA JPL Horizon reports 3- $\sigma$ uncertainties in the position of 0.45 and 0 .' $^{\varsigma} 10$ in RA and Dec, respectively. The measured very marginal differences may be attributed to non-gravitational forces from the distant activity of the comet.

For the photometry, we used local comparison stars taken from the USNO-B catalog. To check the consistency of the magnitude zeropoint (hereafter ZP), another method was also used, based on synthetic $R_{C}$ magnitudes from 2MASS photometry. To calculate the synthetic $R_{C}$ magnitudes, we collected 2MASS photometry of all stars in the Landolt (1992) cata$\log$ and followed the method of least squares to fit the color indices (see Bilir et al. 2008, who give transformations from 2MASS magnitudes to Sloan $g-r$ and $r-i$ colours; we followed the same procedure to determine a transformation from 2MASS magnitudes to $R_{C}$ magnitude). We excluded all stars with $K>13$ from the fitting, and also required that $J-K<0.4$ where the color-color distributions were narrow and linear. We then determined

$R_{C}=J-0.766(J-H)+2.364(J-K)+0.213 \pm 0.055$,

which is valid for stars $(J-K<0.4)$. We identified 15 appropriate stars in our images that had 2MASS photometry. Equation (1) was applied to these stars, and finally the photometric $\mathrm{ZP}$ of the average of $180 \mathrm{~s}$ images inferred a brightness of $24 \mathrm{~m} .42 \pm 0 \mathrm{~m} 06 \mathrm{mag}$. This result is absolutely consistent with a ZP of $24 \mathrm{~m} .45 \pm 0.05$, derived from the $R$ photometry presented in the USNO-B catalog. The $4 \%$ difference between the two different ZPs is much smaller than the photon noise of the comet image itself. We finally adopted a zero-point of 24.43 mag at the average airmass of $\approx 2$ where the observations were taken. This ZP is consistent with the standard value of WFI of $\approx 24.4-24.6$ in the Zenith (chip \#6), and the average $R$ extinction coefficient of $0.07-0.12$.

The comet and the comparison stars were measured in an aperture of 2'.9 diameter, i.e. 1.5-times the $F W H M$ of stellar profiles. This is quite close to the aperture of the optimal $S / N$ $(1.3 \times F W H M$, Szabó et al. 2001b), and contains $85 \%$ of the starlight. The total photon noise (star+background) was 0.08 (1- $\sigma$ confidence interval). Because the comparison stars were measured with the same aperture, there was no need to perform aperture correction. Estimating conservatively an error of $\approx 0$. 1 in both ZP and in the systematics from aperture effects, the total error results to be 0.2 . Surface photometry has been applied to the each pixels of the raw "comet" image, i.e. without deconvolution to the seeing. Errors were estimated based on the background scatter of 1.27 ADU/pixel, or $0.07 \mathrm{ADU} / \mathrm{arcs}^{2}$. The surface brightness profile is plotted in Fig. 2. 


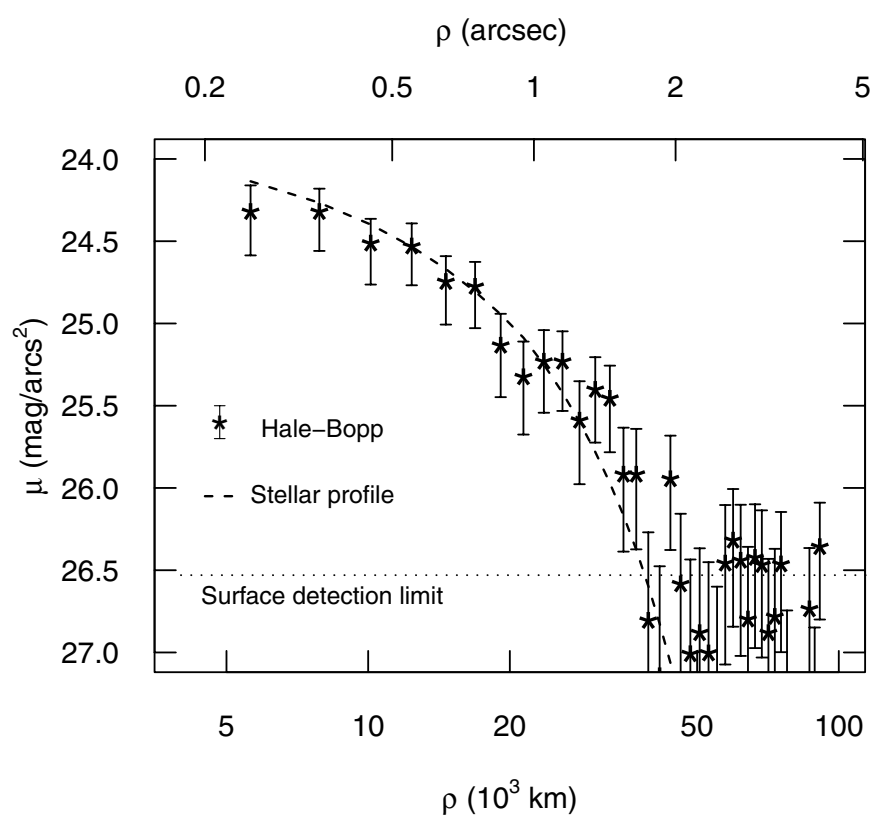

Fig. 2. Surface brightness profile of Hale-Bopp (stars), compared to the average profile of faint stars (dashed line). The dotted line shows the $2-\sigma$ limit of surface brightness detection in the comet image.

\section{Results}

Compared to our previous observations, the general appearance of the comet has drastically changed in the past 3 years. The measured total magnitude of Hale-Bopp was $R=23.3 \pm 0.2$ in 2010, referring to an absolute brightness of $m_{R}(1,1,0)=8$. 3 . This brightness can be converted to $a_{R} C$, the total reflecting surface in the aperture (Eddington 1910)

$a_{R} C=\frac{2.22 \times 10^{22} \pi R^{2} \Delta^{2} 10^{0.4\left(m_{\odot}-m_{\text {comet }}\right)}}{10^{-0.4 \alpha \beta}}$,

where $m_{\odot}=-27 \mathrm{~m} 11$, the apparent $R_{C}$ brightness of the Sun, and the $\beta$ phase coefficient is usually assumed to be 0.04 . Substituting the measured total brightness yields $a_{R} C \approx$ $485 \mathrm{~km}^{2}$. This value is only $11 \%$ of the value measured in late 2007, when Hale-Bopp was at a 25.7 AU solar distance.

Unlike most comets with distant activity, the inner coma of Hale-Bopp followed a distinct, flat profile (Szabó et al. 2008). In 2010, we did not detect such a coma, and the profile of Hale-Bopp was not broader than that of the stars (Fig. 2). This indicated the lack of a bright, extended inner coma. The measured intensity peak of the comet had an apparent surface brightness of $24.3 \mathrm{mag} / \mathrm{arcs}^{2}$. For comparison, the peak intensity was $20.6 \mathrm{mag} / \operatorname{arcs}^{2}$ three years before, and at that time a flat intensity plateau extended to $\approx 2$.' 5 from the nucleus. By late 2010 , the bright flat coma had vanished, suggesting that the matter production in 2010 was at least an order of magnitude less than in 2007. This estimate is also compatible with the measured decrement of $a_{R} C$ by an order of magnitude. In 2010, the comet also lacked coma or tail extending farther than 2 ".5 and exceeding $26.5 \mathrm{mag} / \mathrm{arcs}^{2}$ surface brightness (which is the limit of a $2-\sigma$ detection of extended sources above the background noise). Since a coma-like feature is not present in the images, we conclude that Hale-Bopp had a very faint outer coma, if any, at 30.7 AU solar distance.

Because a value for $a_{R} C$ of $485 \mathrm{~km}^{2}$ contradicts expectations based on previous size estimates, we cannot exclude that a loose coma component was still present around Hale-Bopp at

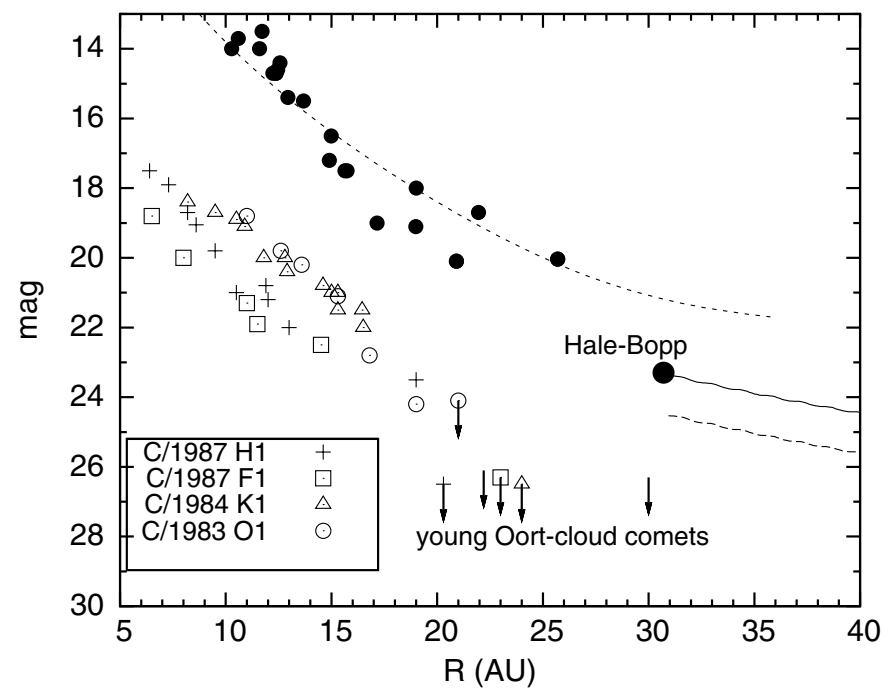

Fig. 3. The light curve of Hale-Bopp compared to observations of six dynamically young Oort-cloud comets (Meech et al. 2004; the last five point are upper limits). The top dashed curve shows the prediction of distant activity by Capria et al. (2002). The middle solid curve is the brightness prediction if the nucleus is already dormant. The lower dashed curve plots the expected brightness of a $37 \mathrm{~km}$ size nucleus (radius) with $4 \%$ albedo (Lamy et al. 2004).

the time of observation. The value of $a_{R} C$ would refer to a total cross-section of $12000 \mathrm{~km}^{2}$ of the reflecting matter, assuming a $4 \%$ albedo. A bare nucleus would be consistent only with this value if it were as large as $62 \mathrm{~km}$ (radius), which is incompatible to the previous size estimates (e.g. $35 \mathrm{~km}$, Weaver \& Lamy 1997; 30 km, Fernández 2003; 37 km, Lamy et al. 2004). Therefore, some part of the reflecting matter must be present in the form of a loose coma. This may be indirect evidence of low-level activity of Hale-Bopp, beyond the orbit of Neptune. Another possibility is that the comet is already dormant, but there might still be dust grains around the nucleus. If there is plenty dust in the aperture, this will contribute significantly to the total light.

In Fig. 3, we plot the observed brightness of Hale-Bopp (collected from ICQ and MPC bulletins) against the solar distance. For comparison, data for six dynamically young Oort-comets are also plotted (Meech et al. 2004). We also show a theoretical light curve predicted from the $\mathrm{CO}$ production curve by Capria et al. (2002), assuming water ice crystallization heating the nucleus. In the case of previous observations, Hale-Bopp was consistently 3-5 mag brighter than these Oort-comets at distances $<15$ AU. By 2010, the total brightness of the comet had diminished by 1.5-2 mag. The observed sudden decrease in the total brightness, together with the disappearance of the prominent coma shows that Hale-Bopp is now on its way to freezing out, i.e. reaching the cessation of matter production.

\section{Discussion and summary}

Our observation is worth comparing to the observations of 1P/Halley at large heliocentric distances (Hainaut et al. 2004). Observed at 28.1 AU, comet Halley had been the most distant comet ever observed until our present observations of Hale-Bopp. The comet Halley was observed with the VLT for an integration time of almost $9 \mathrm{~h}$, leading to the detection of Halley's nucleus with $S / N=8$. At that time, comet Halley was a 28.1 mag atmosphereless body. The significant difference between these comets (in addition to Hale-Bopp being larger and 
brighter) is in the distance out to which comet Hale-Bopp could maintain an observable matter production. The activity of comet Halley ceased at around an 11 AU solar distance. Interestingly, an outburst was observed in 1991 when comet Halley suddenly brightened 5 mag, when the comet was at around 14.2 AU solar distance (West et al. 1991).

In Fig. 3, two curves illustrate the expected brightness of Hale-Bopp beyond 30 AU. The brighter state (solid line) is the predicted light curve if the nucleus is already inactive. (This is a light curve of a body with an identical absolute brightness as Hale-Bopp in late 2010.) The fainter scenario represents the model of Lamy et al. (2004), where we plot the brightness of a body with a $37 \mathrm{~km}$ radius and $4 \%$ albedo. Hale-Bopp was evidently brighter than this latter curve, which we invoked as indirect evidence of a loose coma. However, there is an alternative explanation of the measured brightness. A bare nucleus of $35 \mathrm{~km}$ radius can exhibit the observed brightness if it has a higher albedo, approximately $13 \%$. This is significantly higher than the albedo of cometary nuclei in general and that of derived in particular for Hale-Bopp (4\%, Lamy et al. 2004). However, for there to have been a re-condensation of the gases onto the surface during the distant activity, the surface would have to have been covered with a thin ice film that could increase the albedo. In the case of this scenario, the light curve of Hale-Bopp will follow the path of our brighter prediction. If the measured excess light is due to low-level activity, the light curve will converge to the fainter prediction in the future.

In Szabó et al. (2008), we estimated the basic parameters of the activity of Hale-Bopp at 25.7 AU solar distance. We estimated that its temperature was $53.1 \mathrm{~K}$. This was slightly less than $54.8 \mathrm{~K}$ for a blackbody, because of the sublimation of $2 \times$ $10^{19} \mathrm{CO}$ molecules $/ \mathrm{m}^{2} / \mathrm{s}$. At a $30.7 \mathrm{AU}$ solar distance, the equilibrium temperature of a blackbody is $50.1 \mathrm{~K}$. Since Hale-Bopp seems to turn into an inactive state, we infer that the temperature of activity cessation is somewhere in the range $50-53 \mathrm{~K}$ for a Hale-Bopp type comet.

The main results of this paper can be summarized as follows:

1. We have detected the comet Hale-Bopp at $30.7 \mathrm{AU}$, which is the most distant detection of a comet so far.

2. The absolute brightness of Hale-Bopp was $R(1,1,0)=8 \mathrm{~m} \cdot 3$. The total relative reflecting surface of the $40000 \mathrm{~km}$ environment around the nucleus, $a_{R} C$ is $485 \mathrm{~km}^{2}$. This is a factor of nine less than we observed in 2007, and suggests that the comet is close to ceasing its activity. However, this crosssection is somewhat larger than one would expect for a nucleus with $30-40 \mathrm{~km}$ radius (unless its albedo is very high), so we suggest that some light is scattered by a faint coma, which is still present.
3. The comet has a star-like appearance, hence we conclude that the comet lacks the thick coma that has been characteristic of this comet at all observed solar distances. This situation will finally enable a reliable coma-nucleus separation and the photometry of the nucleus with large telescopes such as VLT.

Acknowledgements. This project has been supported by the Hungarian OTKA Grants K76816, K83790 and MB08C 81013, the "Lendület" Program of the Hungarian Academy of Sciences, and the Eötvös Fellowship of the Hungarian State.

\section{References}

A'Hearn, M. F., Schleicher, D. G., Millis, R. L., Feldman, P. D., \& Thompson, D. T. 1984, AJ, 89, 579

Bilir, S., Ak, S., Karaali, S., et al. 2008, MNRAS, 384, 1178

Capria, M. T., Coradini, A., \& De Sanctis, M. C. 2002, EMP, 90, 217

Caldwell, J. A. R., Cousins, A. W. J., Ahlers, C. C., van Wamelen, P., \& Maritz, E. J. 1993, SAAO Circ, 15, 1

Delsemme, A. H. 1981, in Comets, ed. L. L. Wilkening (Tucson: Univ. Arizona Press), 85

Eddington, A. S. 1910, MNRAS, 70, 442

Fernández, J. A. 2005, Comets (Dordrecht, NL: Springer), Astrophys. Space Libr.

Hainaut, O. R., Delsanti, A., Meech, K. J., \& West, R. M. 2004, A\&A, 417, 1159

Jewitt, D. 2009, AJ, 137, 4296

Landolt, A. U. 1992, AJ, 104, 340

Ivanova, O. V., Skorov, Y. V., Korsun, P. P., Afanasiev, V. L., \& Blum, J. 2011, Icarus, 211, 559

Lamy, P. L., Tóth, I., Fernández, Y. R., \& Weaver, H. A. 2004, in Comets II, ed. M. C. Festou, H. U. Keller, \& H. A. Weaver (Tucson: University of Arizona Press), 223

Lowry, S. C., \& Fitzsimmons, A. 2001, A\&A, 365, 204

Lowry, S. C., \& Fitzsimmons, A. 2005, MNRAS, 358, 641

Lowry, S. C., \& Weissman, P. R. 2003, Icarus, 164, 492

Lowry, S. C., Fitzsimmons, A., Cartwright, I. M., \& Williams, I. P. 1999, A\&A, 349, 649

Mazzotta Epifani, E., Palumbo, P., Capria, M. T., et al. 2006, A\&A, 460, 935

Mazzotta Epifani, E., Palumbo, P., Capria, M. T., et al. 2007, MNRAS, 381, 713

Mazzotta Epifani, E., Palumbo, P., Capria, M. T., et al. 2009, A\&A, 502, 355

Meech, K. J., Buie, M. W., Samarasinha, N. H., Mueller, B. E. A., \& Belton, M. J. S. 1997, AJ, 113, 844

Meech, K. J., Hainaut, O. R., \& Marsden, B. G. 2004, Icarus, 170, 463

Rousselot, P. 2008, A\&A, 480, 543

Snodgrass, C., Lowry, S. C., \& Fitzsimmons, A. 2006, MNRAS, 373, 1590

Snodgrass, C., Lowry, S. C., \& Fitzsimmons, A. 2008, MNRAS, 385, 737

Szabó, Gy. M., Csák, B., Sárneczky, K., \& Kiss, L. L. 2001a, A\&A, 374, 712

Szabó, Gy. M., Csák, B., Sárneczky, K., \& Kiss, L. L. 2001b, A\&A, 375, 285

Szabó, Gy. M., Kiss, L. L., Sárneczky, K., \& Sziládi, K. 2002, A\&A, 384, 702

Szabó, Gy. M., Kiss, L. L., \& Sárneczky, K. 2008, ApJ, 677, L121

Weaver, H. A., Feldman, P. D., A'Hearn, M. F., \& Arpigny, C. 1997, Science, 275,1900

West, R. M., Hainaut, O., \& Smette, A. 1991, A\&A, 246, 77 